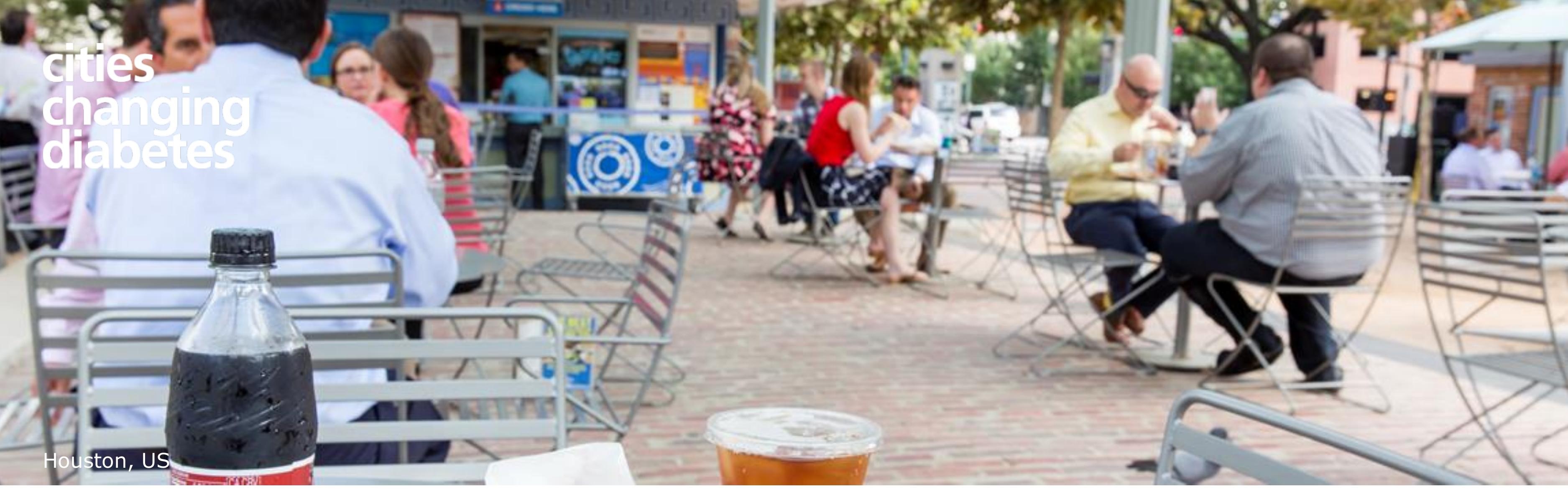

\title{
A NOVEL UNDERSTANDING OF OBESITY AND DIABETES POPULATION DYNAMICS IN CITIES
}

Bagger $M^{1}$, Napier $D^{2}$, Jensen $B^{3}{ }^{3}$, Moses $A^{4}$, Lund $N^{1}$

: Health Advocacy, Novo Nordisk A/S, Denmark; 2: Department of Anthropology, University College London, United Kingdom;

\section{INTRODUCTION}

Diabetes - especially type 2 diabetes (T2D) - is increasing at an alarming rate. However, many cases can be prevented or at least delayed ${ }^{1}$. T2D is a complex disease influenced by various factors and long delays between causes and effects. The global rise is driven primarily by population growth and ageing, physical inactivity, rising overweight and obesity ${ }^{2}$ as well as unhealthy diet ${ }^{3}$. The most significant modifiable driver is excess bodyweight ${ }^{4}$. Worldwide, obesity has more than doubled since $1980^{5}$. In $2014,39 \%$ of adults or more than 1.9 billion adults were overweight - of these more than 600 million were obese equivalent to $13 \%$ of all adults ${ }^{5}$.

\section{RESEARCH AIM}

The aim was to develop a model - the Diabetes Projection Model (DPM) - illustrating how the future diabetes prevalence will be affected by the concomitant obesity level. To illustrate this, two scenarios were designed:

Scenario 1 illustrates how much the diabetes prevalence will increase in the future if the current obesity level is extrapolated linearly.

- Scenario 2 illustrates how much the current obesity level should be reduced in the future to stabilise the diabetes prevalence.

\begin{abstract}
METHOD
Population data for all countries in the world were obtained from the Non-communicable Disease Risk Factor Collaboration (NDCRisC.org) ${ }^{6}$. For each country, population was divided into 15 age groups. From 1975-2014, the population in each age group was divided into seven BMI categories ${ }^{7}$. For each country and age group, share of people in each BMI class was projected depending on scenario. Diabetes risk for each age and BMI group was applied ${ }^{8,9}$ allowing estimations of diabetes prevalence for each country each year. The prevalence for each country was calibrated to match International Diabetes Federation's regional estimates ${ }^{1}$ thereby taking into account differences in way of life, nutrition and genetic disposition for diabetes.
\end{abstract}

\section{RESULTS}

DPM illustrates that the global diabetes prevalence will reach $11.7 \%$ by 2045 and $13.3 \%$ in 2100 if current global obesity rate increases linearly. In contrast, stabilising the global diabetes prevalence at $10.0 \%$ by 2045 will require global obesity rate to be decreased by $25 \%$ by 2045 compared to 2017 . The global diabetes prevalence will even decrease to $9.7 \%$ by 2100 . DPM and the global results have been validated by external experts in obesity and diabetes. Obesity and diabetes population dynamics for a given city can be obtained by feeding in local data. DPM is available online as part of the Urban Diabetes Toolbox at www. citieschangingdiabetes.com.

\section{CONCLUSION}

DPM is simple to use and illustrates what it will take to stabilise the diabetes prevalence. It is designed to help cities set concrete goals for future obesity and diabetes prevalence. It highlights the need for implementing more effective public health strategies targeting obesity.

\section{URBAN DIABETES}

- $54 \%$ of world's population live in urban areas - this is set to increase to $66 \%$ by $2050^{10}$

- $65 \%$ of people with diabetes live in urban areas - this is set to increase to $74 \%$ by 20401 .

\section{CITIES CHANGING DIABETES}

AIM: to map the diabetes challenge, share knowledge, and drive actions in cities.

- SET UP: Novo Nordisk A/S, Steno Diabetes Center Copenhagen, University College London.

\section{GLOBAL DIABETES PREVALENCE BASED ON TWO SCENARIOS}

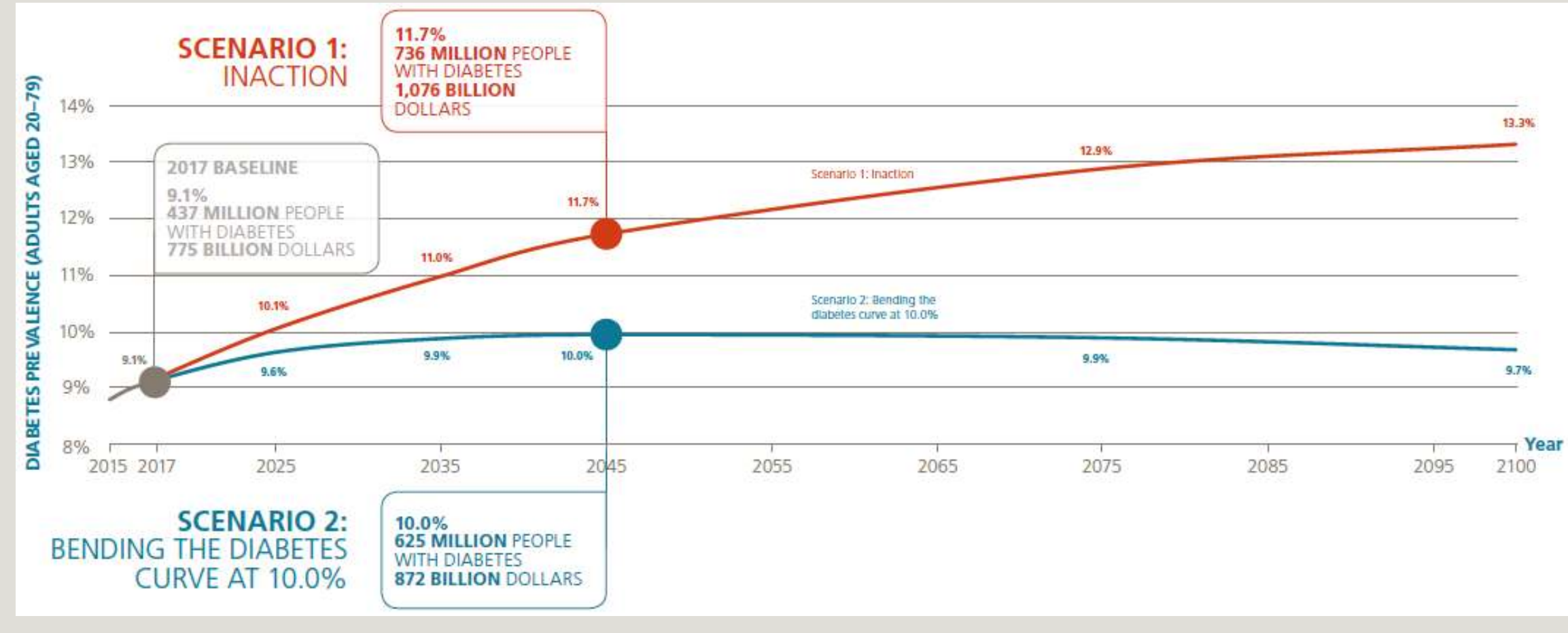

